\title{
AVALIAÇÃO DO EQUILÍBRIO EM ATLETAS DE JIU JITSU, POR MEIO DA PLATAFORMA DE EQUILÍBRIO DO NINTENDO WII
}

\author{
EVALUATION OF EQUILIBRIUM IN JIU JITSU ATHLETES WITH \\ NINTENDO WII BALANCE PLATFORM
}

\author{
Luiz Carlos Alves Junior', Maria Isabel Veras Orselli², \\ Lilian Oliveira de Oliveira ${ }^{3}$, João Rafael Sauzem Machado ${ }^{4}$, \\ Luiz Fernando Rodrigues Junior ${ }^{5}$ Jaqueline de Fátima Biazus ${ }^{6}$
}

\section{RESUMO}

Introdução e objetivos: Brazilian Jiu Jitsu (BJJ) utiliza técnicas de estrangulamento e chaves articulares, nas quais o equilíbrio é uma das aptidões físicas mais imprescindíveis nas atividades dinâmicas adaptativas comumente utilizadas durante os ataques ao adversário. O objetivo do estudo foi analisar o equilíbrio semiestático em praticantes experientes e iniciantes de BJJ. Materiais e Métodos: a amostra foi constituída de 10 atletas do sexo masculino, na faixa etária entre 20 a 40 anos, com graduações diferentes (peso e graduação) e com no mínimo dois anos de treinamento. Foram excluídos os atletas que apresentaram lesões musculares, cardíacas, neurológicas e ósseas que impossibilitem a realização dos testes propostos. O protocolo proposto caracterizou-se na análise do equilíbrio por meio do Nintendo Wii Balance Board (WBB), com o objetivo de analisar e mensurar os graus de equilíbrio postural por meio das oscilações posturais na posição ortostática nas direções látero-lateral e ântero-posterior (AP), com olhos abertos e fechados, cada tentativa teve 60 segundos de duração. Resultados: os resultados demostraram que não houve variação significativa dos valores dessas variáveis espaciais com o tempo de treinamento, mas teve o decrescimento da velocidade de oscilação AP com o tempo de treinamento. Conclusão: observa-se que o tornozelo está exposto a uma força que afeta o controle do equilíbrio nos atletas de BJJ avaliados, na condição BOA (olhos abertos) mais do que na BOF (olhos fechados). Atletas mais experientes exibiram maior controle do equilíbrio postural estático de olhos abertos do que em atletas iniciantes na direção ântero-posterior.

Palavras-chave: Equilíbrio postural, Atletas, Avaliação.

\section{ABSTRACT}

Introduction and objectives: Brazilian Jiu Jitsu (BJJ) uses strangulation techniques and joint keys, where balance is one of the most essential physical skills in the adaptive dynamic activities commonly used during attacks on the opponent. The objective of the study was to analyze the static balance in experienced and beginner practitioners of BJJ. Materials and Methods: The sample consisted of 10 male athletes in the age group between 20 and 40 years old, with different grades (weight and range) and with at least 2 years of training. The proposed protocol was characterized in the balance analysis on the Nintendo Wii Balance Board

\footnotetext{
${ }^{1}$ Acadêmico do Curso de Fisioterapia - Universidade Franciscana - UFN. E-mail: luizcaj2014@gmail.com

${ }^{2}$ Colaboradora. Dra. Docente do Curso de Engenharia Biomédica - Universidade Franciscana - UFN. E-mail: mbebelveras@ gmail.com

${ }^{3}$ Colaboradora. Dra. Docente do Curso de Fisioterapia - Universidade Franciscana - UFN. E-mail: licafisiot@hotmail.com

${ }^{4}$ Colaborador. MsC. Docente do Curso de Fisioterapia - Universidade Franciscana - UFN. E-mail: drjoaorafa@yahoo.com.br

${ }^{5}$ Colaborador. Dr. Docente do Curso de Engenharia Biomédica - Universidade Franciscana - UFN. E-mail: luizfrjr@ gmail.com

${ }^{6}$ Orientadora. MsC. Docente do Curso de Fisioterapia - Universidade Franciscana - UFN. E-mail: jaquebiazus@hotmail.com
} 
$W B B$, with the objective of analyzing and measuring the degrees of postural balance through the postural oscillations in the orthostatic position in the lateral and anteroposterior (AP) directions, with eyes opened and closed, each attempt was 60 seconds long. Results: The results showed that there was no significant variation of the values of these spatial variables with the training time, but the AP oscillation velocity decreased with the training time. It is observed that the ankle is exposed to a force that affects the balance control in the BJJ athletes assessed, in the BOA condition (open eyes) rather than in the BOF (closed eyes). Conclusion: More experienced athletes exhibited greater control of open-balance static posture balance than in beginner athletes in the anteroposterior direction.

Keywords: Postural Balance, Athletes, Evaluation.

\section{INTRODUÇÃO}

O Brazilian Jiu Jitsu (BJJ) é uma categoria de confronto esportivo que tem por finalidade a subjugação do oponente, especialmente por meio de técnicas específicas de estrangulamentos e chaves articulares, tendo como base as alavancas articulares (MOREIRA et al., 2012). O tempo de combate varia de acordo com as suas graduações, com menores durações para faixas brancas (cinco minutos) e maiores para faixas pretas (10 minutos) (BRASIL et al., 2015), tendo como característica esforços inconstantes, com variações rotineiras de forças de maiores e menores intensidades (ANDREATO et al., 2013) com uma relação de esforço/pausa de 6:1 ou 8:1 (ANDREATO et al., 2015).

O conhecimento processual referente ao equilíbrio é uma das aptidões físicas mais imprescindíveis para os atletas praticantes de artes marciais, como o BJJ (AOYAMA et al., 2017). O equilíbrio se dá com um bom controle do centro de gravidade e da distribuição corporal, principalmente nas atividades dinâmicas adaptativas comumente utilizadas durante os ataques ao adversário. Com o centro de pressão (COP) se deslocando de uma forma equilibrada e o sistema nervoso central controlando os movimentos por meio do centro de massa do corpo (COM), haverá um maior equilíbrio semiestático, favorecendo um melhor desempenho do atleta (BRASIL et al., 2015; TAKEDA et al., 2017).

A melhor análise direta referente ao equilíbrio se dá pela avaliação do deslocamento do COP, sendo o padrão-ouro em avaliação de equilíbrio semiestático, por identificar variações suaves não encontradas por outras avaliações subjetivas. A avaliação do centro de pressão (COP) pode ser feita de forma indireta por meio das plataformas de força, como o Nintendo Wii Balance Board (WBB, Nintendo of America Inc, Redmond, WA), apresentando-se como uma opção portátil, de custo reduzido e com elevado padrão de validade e confiabilidade (ROCHEFORT et al., 2017; HUURNINK et al., 2013; BORNA et al., 2017).

Este estudo justifica-se pela possibilidade de se ter um método de avaliação portátil e com um alto grau de confiabilidade (WEAVER; MA; LAING, 2017), sendo a portabilidade uma característica-chave na maioria dos contextos, como em organizações não profissionais e em instituições de ensino 
superior (SEVERINE et al., 2017). A utilização desse instrumento visou buscar parâmetros para a melhora da performance e da aplicabilidade do golpe, resultando no aprimoramento do padrão luta.

Os objetivos do presente estudo foram: avaliar o equilíbro de atletas praticantes de Jiu Jitsu, analisar a amplitude e áreas de oscilação ântero-posterior e látero-lateral, bem como avaliar a velocidade de oscilação nas direções ântero-posterior e látero-lateral.

\section{MATERIAIS E MÉTODOS}

Este estudo consistiu em uma pesquisa quantitativa, do tipo quase experimental. O experimento foi conduzido no Laboratório de Ensino Prático em Fisioterapia (LEP) da Universidade Franciscana (UFN), localizada em Santa Maria (RS). Para realização da pesquisa, houve a aprovação do Comitê de Ética em Pesquisa sob o n 2.432 .537 . Teve seu desenvolvimento no primeiro semestre de 2018, no período de fevereiro a junho.

Esta pesquisa respeitou ou aspectos éticos, de acordo com a Resolução 466/12 e complementares - Conselho Nacional de Saúde. Foi realizado um prévio contato com as academias para explicar os termos da pesquisa e convidar os atletas e o treinador a participarem. Critérios de inclusão: não apresentarem lesões musculares e ósseas no momento da avaliação; estarem dentro da faixa etária descrita; terem o tempo de treinamento pré-estabelecido; terem disponibilidade de horário e assinarem o Termo de Consentimento Livre e Esclarecido. Critérios de exclusão: apresentarem lesões musculares, cardíacas, neurológicas e ósseas que impossibilitem a realização dos testes propostos; não estarem realizando treinamento há mais de uma semana.

Foram utilizados como instrumentos de avaliação: uma ficha de avaliação composta por questões abertas e fechadas contendo dados pessoais, medicamentos porventura utilizados, histórico de fraturas, tempo de treinamento, tempo de afastamento do treino, graduação, e a plataforma Nintendo Wii Balance Board (WBB): a Nintendo Wii Balance Board (WBB, Nintendo of America Inc, Redmond, WA), um instrumento para analisar e mensurar graus de equilíbrio postural por meio das oscilações posturais na posição ortostática nas direções látero-lateral e ântero-posterior (BORNA et al.; 2017).

Os atletas foram instruídos a posicionarem-se em cima da plataforma de pressão em posição ortostática e com os pés distanciados na largura do quadril para a calibração da plataforma. Durante o teste, o atleta permaneceu em pé com a cabeça voltada para frente em duas condições: olhos abertos (OA) com foco fixado em um alvo a uma distância de aproximadamente 2 m; olhos fechados (OF) com braços ao longo do corpo, sem calçados. Foram feitas três tentativas em cada condição (OA e OF), totalizando seis tentativas para cada atleta, entre uma tentativa e outra houve um intervalo de 30 segundos. A taxa de amostragem da plataforma foi de $30 \mathrm{~Hz}$ e o tempo de aquisição, 60 segundos. 
Para analisar os resultados dos testes quantitativos, foi utilizado o Coeficiente de Pearson para avaliar a correlação entre o tempo de treinamento e as variáveis de equilíbrio. As análises estatísticas foram realizadas utilizando-se o software Matlab (MathWorks Inc., Natick, MA, USA) e foi considerado Alpha $=0,05$ para o nível de significância dos resultados.

\section{RESULTADOS}

A população foi constituída de 20 atletas adultos, do gênero masculino da cidade de Santa Maria (RS), com graduações diferentes (peso e graduação), praticantes de Jiu Jitsu com no mínimo dois anos de treinamento e na faixa etária entre 20 a 40 anos.

Dos 20 atletas que estavam dentro dos critérios de inclusão, houve uma perda amostral de nove atletas, decorrente de um problema de funcionamento da plataforma WBB.

Destes 11 que realizaram a avaliação inicial, um atleta, durante o acompanhamento dos treinos relatou lesão, sendo excluído da amostra final.

Restando assim 10 atletas de competição com média de idade de 31,2 anos; média de peso 86 kg; média de altura 177,8 cm; média de tempo de treinamento quatro anos. Dos 10 atletas, 100\% são destros em membro inferior. A caracterização da amostra consta na tabela 1.

Tabela 1 - Caracterização da amostra.

\begin{tabular}{cccccc}
\hline Identificação & $\begin{array}{c}\text { Anos de } \\
\text { idade }\end{array}$ & Peso $(\mathbf{k g})$ & Altura (cm) & $\begin{array}{c}\text { Tempo de } \\
\text { Treinamento }\end{array}$ & $\begin{array}{c}\text { Membro } \\
\text { Dominante }\end{array}$ \\
\hline CV & 30 & 95 & 177 & 4 anos & MID \\
\hline DK & 32 & 82 & 176 & 6 anos & MID \\
FA & 23 & 76 & 185 & 4,5 anos & MID \\
\hline GD & 30 & 117 & 183 & 2 anos & MID \\
JM & 27 & 67 & 169 & 3 anos & MID \\
\hline LC & 38 & 91 & 186 & 2 anos & MID \\
LV & 37 & 66 & 170 & 2 anos & MID \\
MM & 31 & 74 & 179 & 8 anos & MID \\
RL & 29 & 72 & 171 & 6 anos & MID \\
\hline TB & 35 & 120 & 182 & 2,5 anos & MID \\
\hline
\end{tabular}

Fonte: elaborada pelo autor (2018).

Os atletas que fizeram parte do estudo treinam em quatro academias diferentes. Observou-se que, durante os treinamentos, não houve um padrão de treino e intensidade. Não houve variação significativa dos valores das variáveis espaciais com o tempo de treinamento, como é possível observar na relação do gráfico 1 com o gráfico 2 . 
Gráfico 1 - Relação das variáveis espaciais, deslocamento total, desvio padrão em cada direção e a área de oscilação em função do tempo de treinamento em anos na condição de apoio bipodal com olhos abertos.
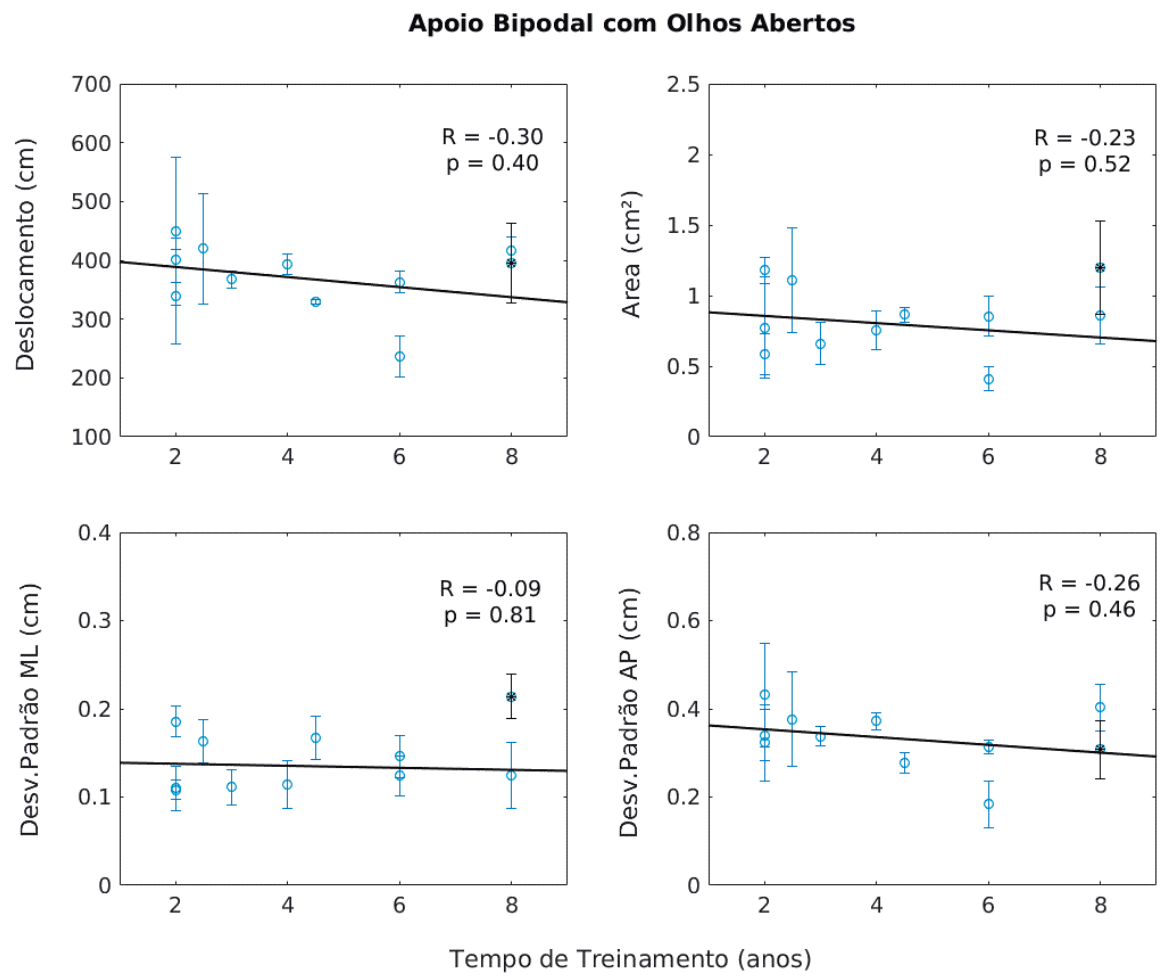

Legenda: Em azul está o valor médio das três tentativas (pontos) e o desvio padrão (“antenas") de cada sujeito. Em preto está a reta que indica como cada variável se comporta com o tempo de treinamento. O ponto com asterisco foi o sujeito desconsiderado na análise devido a lesão pós início de coleta. R é o valor do coeficiente de correlação linear entre as duas variáveis de desvio padrão e o tempo de treino, e indica a quão bem essa reta representa o comportamento dos dados.

Fonte: elaborado pelo autor (2018).

O valor de $\mathrm{p}$ indica a probabilidade de esse modelo (de variação linear) não representar bem os dados. Como para todas as grandezas que descrevem o controle do equilíbrio, tanto na condição de olhos abertos como na condição de olhos fechados, essa probabilidade é alta em relação ao nível de corte (ou seja, $p>0.05$ ). Assim, pode-se dizer que não houve variação significativa dos valores dessas variáveis espaciais com o tempo de treinamento.

A relação do gráfico 3 com o gráfico 4 mostrou que o modelo de variação linear não representa bem o comportamento do conjunto de dados para a velocidade medial lateral tanto no apoio com olhos abertos como no apoio com olhos fechados. Isso indica que não houve nem aumento, nem diminuição importante da velocidade de oscilação MD com o tempo de treinamento. Quando se olha para a velocidade anterior posterior vê-se que ela tem um decrescimento bastante pronunciado (reta) e que o modelo linear e a chance de esse modelo representar bem o decrescimento da velocidade de oscilação AP com o tempo de treinamento é alta para o valor de $\mathrm{p}(\mathrm{p}<0.05)$. 
Gráfico 2 - Relação das variáveis espaciais, deslocamento total, desvio padrão em cada direção e a área de oscilação em função do tempo de treinamento em anos, na condição de apoio bipodal com olhos Fechados
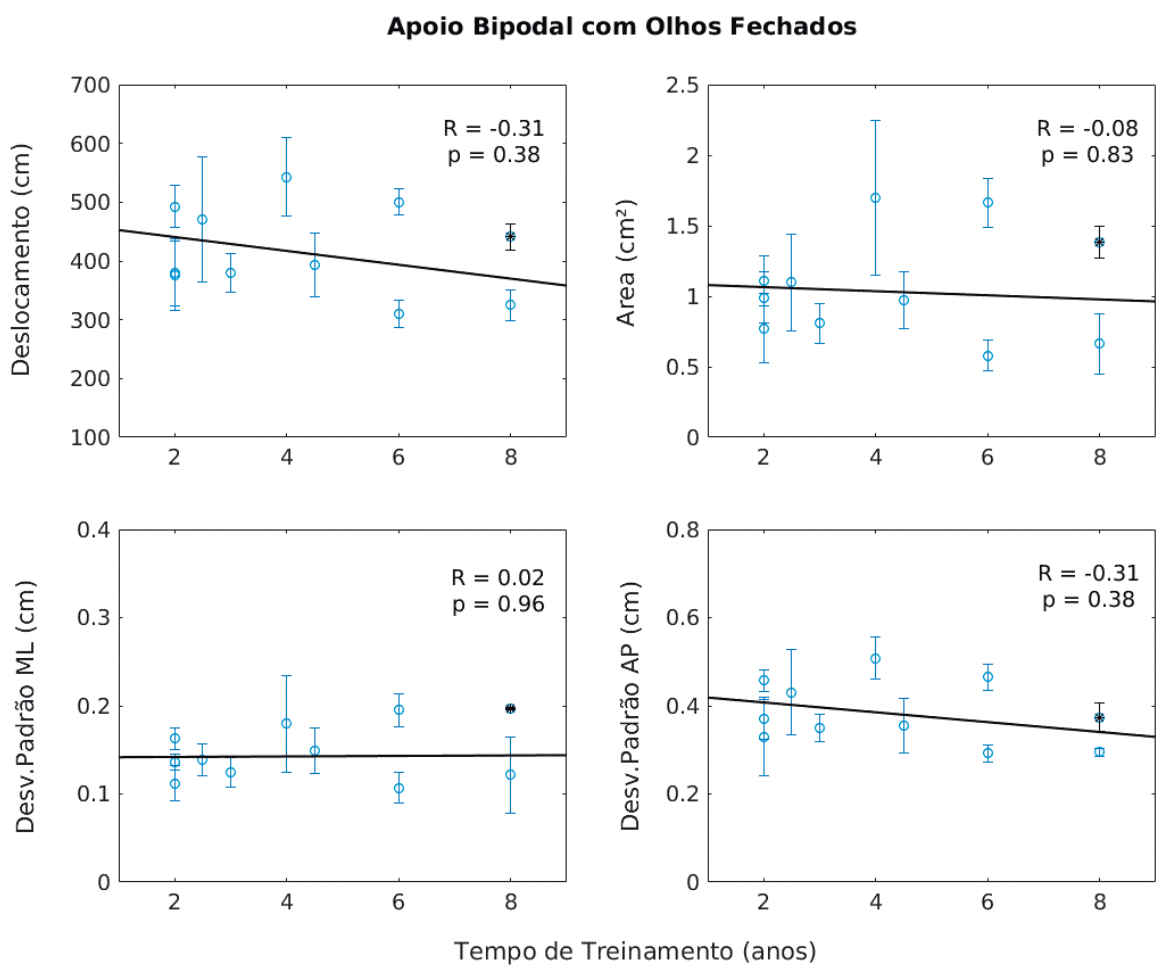

Legenda: Em azul está o valor médio das três tentativas (pontos) e o desvio padrão (“antenas”) de cada sujeito. Em preto está a reta que indica como cada variável se comporta com o tempo de treinamento. O ponto com asterisco foi o sujeito desconsiderado na análise devido a lesão pós início de coleta. R é o valor do coeficiente de correlação linear entre cada variável de velocidade e o tempo de treino, e indica a quão bem essa reta representa o comportamento do conjunto de dados.

Fonte: elaborado pelo autor (2018).

Gráfico 3 - Variáveis de velocidade que se referem aos componentes da velocidade nas duas direções e velocidade resultante em função do tempo de treinamento em anos, na condição de apoio bipodal com olhos abertos
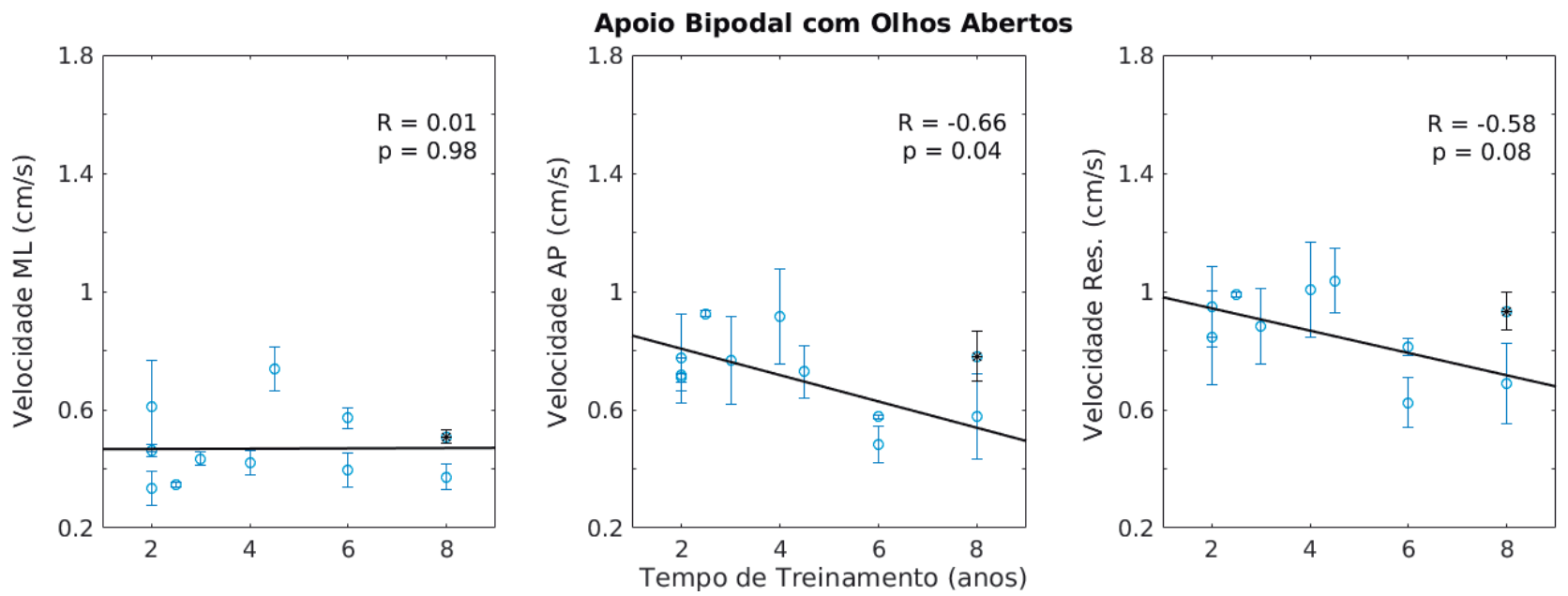

Legenda: Em azul está o valor médio das três tentativas (pontos) e o desvio padrão (“antenas”) de cada sujeito. Em preto está a reta que indica como cada variável se comporta com o tempo de treinamento. O ponto com asterisco foi o sujeito desconsiderado na análise devido a lesão pós início de coleta. R é o valor do coeficiente de correlação linear entre cada variável de velocidade e o tempo de treino, e indica a quão bem essa reta representa o comportamento do conjunto de dados.

Fonte: elaborado pelo autor (2018). 
Gráfico 4 - Variáveis de velocidade que se referem aos componentes da velocidade nas duas direções e velocidade resultante em função do tempo de treinamento em anos, na condição de apoio bipodal com olhos abertos
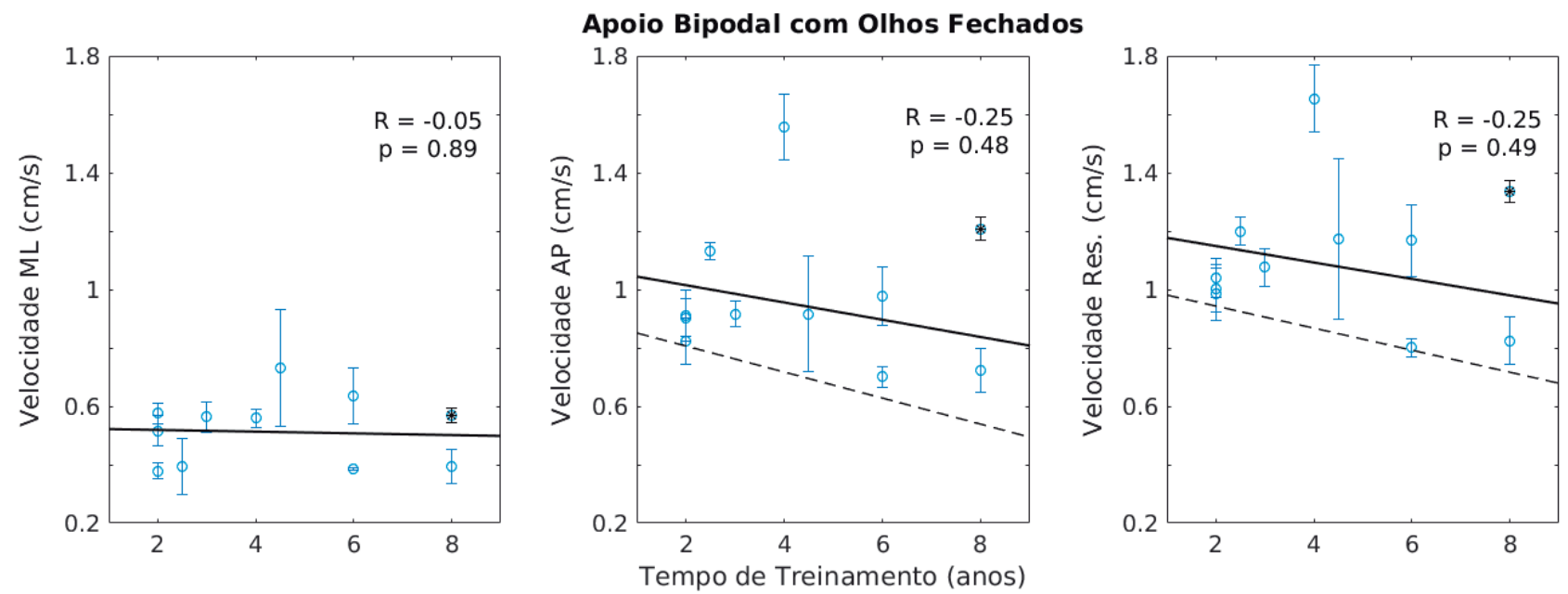

Legenda: Em azul está o valor médio das três tentativas (pontos) e o desvio padrão ("antenas") de cada sujeito. Em preto está a reta que indica como cada variável se comporta com o tempo de treinamento, a linha pontilhada representa a variação da velocidade AP e resultante com tempo. O ponto com asterisco foi o sujeito desconsiderado na análise devido a lesão pós início de coleta. R é o valor do coeficiente de correlação linear entre cada variável de velocidade e o tempo de treino, e indica a quão bem essa reta representa o comportamento do conjunto de dados.

Fonte: elaborado pelo autor (2018).

\section{DISCUSSÃO}

O principal achado do presente estudo foi que os atletas de competição com maior tempo de treinamento do BJJ obtiveram melhores resultados no equilíbrio semiestático na direção ântero-posterior de olhos abertos em comparação a atletas com menor tempo, o que está de acordo com os achados de Dorneles, Silva e Mota (2015), que sugerem que os mecanismos de controle postural não são os mesmos na direção médio-lateral e ântero-posterior. Até o presente momento, não foram encontrados numerosos estudos avaliando o equilíbrio em praticantes experientes de BJJ em comparação a praticantes iniciantes, embora tal capacidade seja fundamental para manutenção do centro de gravidade e relevante para praticantes de Brazilian Jiu Jitsu (BJJ) (MENDONÇA et al., 2017).

Os resultados do presente estudo são similares aos observados na pesquisa de Brasil et al. (2015), realizado com o BJJ, no qual três grupos foram divididos em praticantes experientes, praticantes iniciantes e não praticantes (BRASIL et al., 2015). Os autores observaram que os atletas experientes de BJJ tiveram um melhor controle do equilíbrio comparado aos iniciantes. Para que esse equilíbrio seja obtido, é imprescindível que os sistemas básicos referentes ao equilíbrio: vestibular, visual e proprioceptivo, além de outras funções, transportem informações espaciais para o Sistema Nervoso Central, que organizará esses elementos a fim de controlar a postura corporal (MORAES et al., 2014).

Além disso, as diferenças também podem depender da capacidade dos atletas de usar informações provenientes de outras vias aferentes, principalmente do sistema somatossensorial, para controlar a 
COP (SABCHUK; BENTO; RODACKI, 2012), indo ao encontro dos nossos achados, em que, na condição de olhos fechados, não houve uma diferença significativa entre os atletas experientes e os iniciantes no qual se pode sugerir a importância de um trabalho proprioceptivo durante os treinamentos.

A diferença no conhecimento processual referente ao equilíbrio de olhos abertos entre atletas experientes e aletas iniciantes, verificadas no presente estudo, podem ser explicadas por condições diversas, como o nível de aptidão física ou grau de aprendizagem motora das habilidades específicas da modalidade, pelo fato de o BJJ constituir-se de movimentos nos quais os lutadores sofrem perturbações inesperadas durante o combate, por meio de puxões e empurrões realizados por seus adversários (LIMA et al., 2017). Segundo Brasil et al. (2015), pesquisas recentes encontraram que o controle do centro de gravidade e da disposição do peso corporal em atividades dinâmicas está relacionado a um aumento na execução de atividades que requerem um bom equilíbrio, como o Jiu Jitsu, justificando o maior aperfeiçoamento do equilíbrio de olhos abertos em comparação aos atletas iniciantes.

Entretanto o presente estudo também constatou que a comparação entre atletas experientes e iniciantes de olhos fechados não demostrou comportamento semelhante na condição de olhos abertos, isso sugere que os participantes não tiveram oportunidade de desenvolver estratégias posturais com base nos inputs não visuais durante o tempo de treinamento, indo ao encontro dos resultados encontrados por Junior et al. (2015), que constataram que estímulos de diferentes vias aferentes proporciona uma acessibilidade de adaptação maior e, por consequência menor oscilação corporal, em ocasiões em que ocorre a diminuição ou ausência de informações visuais.

Segundo Bruniera et al. (2015), biomecanicamente, a influência sobre o equilíbrio necessita da manutenção do centro de gravidade (CG) sobre a base de sustentação durante circunstâncias estáticas e dinâmicas do movimento, em que o aperfeiçoamento pode decorrer de diferentes formas, tanto em função da maior disparidade de ocasiões de instabilidade corporais vivenciadas por meio das lutas e treinos (KIM et al., 2018) como em função do aprimoramento do controle motor específico e especialização dos hemisférios cerebrais (DORNELES; SILVA; MOTA, 2015). Desse modo, a discrepância entre os atletas experientes e iniciantes sugere que a quantidade de experiência nas aptidões motoras específicas do BJJ pode afetar a capacidade de equilíbrio.

Assim, o atual estudo indica que o equilíbrio semiestático pode ser modificável entre atletas de BJJ. Além disso, a resistência muscular e isométrica de membros inferiores e superiores, pode sofrer alterações, como indicado por Castro et al. (2016). Na mesma direção, Belém et al. (2016) apontam que artes marciais podem amplificar capacidades posturais específicas. Assim, os movimentos que são exercitados em determinado esporte, como no Jiu Jitsu, no qual são praticadas projeções e estabilizações em diversas posições, podem motivar adequações posturais induzidas pela obtenção de novas habilidades motoras específicas.

No presente estudo, observou-se que a força a que o tornozelo está sujeito de fato afeta o controle do equilíbrio nos atletas de BJJ avaliados, na condição BOA mais do que na BOF. Isso corrobora 
a hipótese de que o aumento do tempo de treinamento e o aumento da força, sozinhos, não melhoram o controle do equilíbrio na privação de informação sensorial. Assim, o treino proprioceptivo é de grande importância.

\section{CONCLUSÃO}

Os resultados do presente estudo sugerem que o nível de experiência dos atletas de competição do BJJ pode influenciar o controle do equilíbrio semiestático. Atletas mais experientes demonstraram maior controle do equilíbrio postural semiestático de olhos abertos do que em atletas iniciantes na direção anteroposterior.

Como limitações, cita-se o reduzido tamanho da amostra, que ficou comprometida devido ao mau funcionamento da WBB. Além disso, não foram avaliadas variáveis de assiduidade de treinamento durante o tempo de prática esportiva, o que poderia apresentar relações com a prática da modalidade de combate ou relacionadas a aspectos específicos da preparação física de atletas de BJJ.

Portanto, sugere-se que futuros estudos examinem as variáveis espaciais, propriocepção e sua possível influência no controle do equilíbrio entre participantes experientes e iniciantes do BJJ

\section{REFERÊNCIAS}

ANDREATO, L. V. et al. Brazilian Jiu-Jitsu Simulated Competition Part II: Physical Performance, Time-Motion, Technical-Tactical Analyses, and Perceptual Responses. Journal of Strength and Conditioning Research, [s. l.], v. 29, n. 7, p. 2015-2025, 2015. Disponível em: https://doi.org/10.1519/ JSC.0000000000000819. Acesso em: 14 dez. 2020.

AOYAMA, Y. et al. Immediate effect of subliminal priming with positive reward stimuli on standing balance in healthy individuals. Medicine (United States), [s. l.], v. 96, n. 28, 2017. Disponível em: https://doi.org/10.1097/MD.0000000000007494. Acesso em: 14 dez. 2020.

BELEM, I. et al. O estresse no MMA: As estratégias de enfrentamento podem melhorar o desempenho dos lutadores? Revista Brasileira de Medicina do Esporte, [s. l.], v. 22, n. 4, p. 287-290, 2016. Disponível em: https://doi.org/10.1590/1517-869220162204147514. Acesso em: 14 dez. 2020.

BRASIL, B. et al. Comparação do equilíbrio dinâmico entre praticantes de Brazilian Jiu-Jitsu com diferentes níveis de experiência. Revista Brasileira de Educação Física e Esporte, [s. l.], v. 29, n. 4, p. 535-541, 2015. Disponível em: https://doi.org/10.1590/1807-55092015000400535. Acesso em: 14 dez. 2020. 
BRUNIERA, J. R. Z. et al. Análise comparativa do equilíbrio postural pela posturografia em pacientes com vertigem isolada ou associada com perda auditiva. Audiology - Communication Research, [s. l.], v. 20, n. 4, p. 321-326, 2015. Disponível em: https://doi.org/10.1590/2317-6431-2014-1534. Acesso em: 14 dez. 2020.

CASTRO, M. L. de et al. Influência de tarefas cognitivas sobre o equilíbrio estático de indivíduos saudáveis. ConScientiae Saúde, [s. l.], v. 15, n. 2, p. 273-280, 2016. Disponível em: https://doi.org/10.5585/ conssaude.v15n2.6463. Acesso em: 14 dez. 2020.

DE MENDONÇA, D. L. C. et al. Assessment of the quality of life, muscle strength, and dynamic balance of elderly kendo players. Clinics, [s. l.], v. 72, n. 11, p. 661-666, 2017. Disponível em: https:// doi.org/10.6061/clinics/2017(11)03. Acesso em: 14 dez. 2020.

DORNELES, P. P.; SILVA, F. S.; MOTA, C. B.. Comparação do equilíbrio postural entre grupos de mulheres com diferentes faixas etárias. Fisioterapia e Pesquisa. São Paulo, v. 22, n. 4, p. 392-397, dez. 2015. Disponível em: http://dx.doi.org/10.590/1809-2950/14270622042015. Acesso em: 14 dez. 2020.

HUURNINK, A. et al. Comparison of a laboratory grade force platform with a Nintendo Wii Balance Board on measurement of postural control in single-leg stance balance tasks. Journal of Biomechanics, [s. l.], v. 46, n. 7, p. 1392-1395, 2013. Disponível em: https://doi.org/10.1016/j.jbiomech.2013.02.018. Acesso em: 14 dez. 2020.

KIM, M. et al. Specific muscle synergies in national elite female ice hockey players in response to unexpected external perturbation. Journal of Sports Sciences, [s. l.], v. 36, n. 3, p. 319-325, 2018. Available at: https://doi.org/10.1080/02640414.2017.1306090. Acesso em: 14 dez. 2020.

LIMA JÚNIOR, J. C.; et al.. Avaliação do equilíbrio estático em indivíduos com estrabismo. Revista Fisioterapia \& Saúde Funcional, Fortaleza, v. 4, n. 1, p. 23-29, jan./jun. 2015. Disponível em: https:// bit.ly/35QHH9e. Acesso em: 14 dez. 2020.

LIMA, P. O. de P. et al. BIOMECHANICAL DIFFERENCES IN BRAZILIAN JIU-JITSU ATHLETES: THE ROLE OF COMBAT STYLE. International journal of sports physical therapy, [s. l.], v. 12, n. 1, p. 67-74, 2017. Disponível em: https://bit.ly/2NaUnBi. Acesso em: 14 dez. 2020.

MERCHANT-BORNA, K. et al. Evaluation of nintendo Wii balance board as a tool for measuring postural stability after sport-related concussion. Journal of Athletic Training, [s. l.], v. 52, n. 3, p. 245-255, 2017. Disponível em: https://doi.org/10.4085/1062-6050-52.1.13. Acesso em: 14 dez. 2020. 
MORAES, A. G. et al. Comparação do equilíbrio postural unipodal entre crianças e adultos. Revista Brasileira de Educação Física e Esporte, [s. l.], v. 28, n. 4, p. 571-577, 2014. Disponível em: https:// doi.org/10.1590/1807-55092014000400571. Acesso em: 14 dez. 2020.

MOREIRA, A. et al. Salivary cortisol and immunoglobulin a responses to simulated and official jiu-jitsu matches. Journal of Strength and Conditioning Research, [s. l.], v. 26, n. 8, p. 2185-2191, 2012. Disponível em: https://doi.org/10.1519/JSC.0b013e31823b8702. Acesso em: 14 dez. 2020.

ROCHEFORT, C. et al. Balance Markers in Adolescents at 1 Month Postconcussion. Orthopaedic Journal of Sports Medicine, [s. l.], v. 5, n. 3, p. 232596711769550, 2017. Disponível em: https://doi. org/10.1177/2325967117695507. Acesso em: 14 dez. 2020.

SABCHUK, R. A. C.; BENTO, P. C. B.; RODACKI, A. L. F. Comparison between field balance tests and force platform. Revista Brasileira de Medicina do Esporte, [s. l.], v. 18, n. 6, p. 404-408, 2012. Disponível em: https://doi.org/10.1590/S1517-86922012000600012

SEVERINI, G. et al. Use of Nintendo Wii Balance Board for posturographic analysis of Multiple Sclerosis patients with minimal balance impairment. Journal of NeuroEngineering and Rehabilitation, [s. l.], v. 14, n. 1, 2017. Disponível em: https://doi.org/10.1186/s12984-017-0230-5. Acesso em: 14 dez. 2020.

TAKEDA, K. et al. Adaptation effects in static postural control by providing simultaneous visual feedback of center of pressure and center of gravity. Journal of Physiological Anthropology, [s. l.], v. 36, n. 1, 2017. Disponível em: https://doi.org/10.1186/s40101-017-0147-5. Acesso em: 14 dez. 2020.

WEAVER, T. B.; MA, C.; LAING, A. C. Use of the Nintendo Wii Balance Board for studying standing static balance control: Technical considerations, force-plate congruency, and the effect of battery life. Journal of Applied Biomechanics, [s. l.], v. 33, n. 1, p. 48-55, 2017. Disponível em: https://doi.org/10.1123/ jab.2015-0295. Acesso em: 14 dez. 2020. 
